\title{
Proposal for the determination of nuclear masses by high-precision spectroscopy of Rydberg states
}

\author{
B J Wundt and U D Jentschura \\ Department of Physics, Missouri University of Science and Technology, Rolla, MO \\ 65409-0640, USA
}

\begin{abstract}
The theoretical treatment of Rydberg states in one-electron ions is facilitated by the virtual absence of the nuclear-size correction, and fundamental constants like the Rydberg constant may be in the reach of planned high-precision spectroscopic experiments. The dominant nuclear effect that shifts transition energies among Rydberg states therefore is due to the nuclear mass. As a consequence, spectroscopic measurements of Rydberg transitions can be used in order to precisely deduce nuclear masses. A possible application of this approach to the hydrogen and deuterium, and hydrogen-like lithium and carbon is explored in detail. In order to complete the analysis, numerical and analytic calculations of the quantum electrodynamic (QED) self-energy remainder function for states with principal quantum number $n=5, \ldots, 8$ and with angular momentum $\ell=n-1$ and $\ell=n-2$ are described $\left(j=\ell \pm \frac{1}{2}\right)$.
\end{abstract}

PACS numbers: 06.20.Jr, 12.20.Ds, 31.30.jf

Submitted to J. Phys. B 


\section{Introduction}

The celebrated high-precision spectroscopic experiments of atomic hydrogen [1, 2, 3, 4, 5, 6 , 7. 8, 9, 10] typically involve transitions among quantum states with low principal quantum numbers, e.g., the hydrogen $1 S-2 S$ transition. Unfortunately, the rather large uncertainty of the root-mean-square (RMS) charge radius of the proton limits the precision to which the nominal accuracy of spectroscopic experiments can be used in order to infer fundamental constants: while the most precisely measured transition in atomic hydrogen is known to an accuracy of 1.8 parts in $10^{14}$, the most accurately determined fundamental constant (the Rydberg) is known only to an accuracy of 6.6 parts in $10^{12}$ (see Ref. [11]). The subject is not without intricacies: Even the correct definition of the RMS charge radius for lowenergy processes in atomic systems is a rather fundamental problem which necessitates a careful study of form factors which are a priori defined for scattering processes [12, 13, 14]. Indeed, in the 2006 adjustment of the fundamental constants [11], the RMS charge radius of the proton and deuteron are inferred by fitting the 23 most accurately measured transitions to QED theory [15], using the method of least squares.

For the low-lying states canonically used for high-precision spectroscopy experiments, the dominant nuclear effect is from the nuclear size effect on the Dirac energy. By contrast, the QED theory of transitions among highly excited states of one-electron ions is simplified because of the virtual absence of the problematic nuclear size effect, and the dominant nuclear proerty that influences the spectrum is the nuclear mass. This effect is well understood, and therefore, the theory of Rydberg transitions among states with high angular momenta can be formulated very precisely. In Refs. [16, 17], Rydberg states have been proposed in order to avoid these limitations and to improve the accuracy of the Rydberg constant. If we are interested in determining the Rydberg constant, then it is important to consider hydrogen-like ions with well-known nuclear masses. Conversely, it becomes possible, using precise QED theory, to infer the nuclear mass from spectroscopic measurements, if that mass is not known to sufficient accuracy by other methods. That latter aspect forms the basis of the current investigation and the current proposal which is being investigated in this work. In selecting suitable atomic Rydberg transitions for a conceivable determination of nuclear masses by this method, it is important to consider frequencies accessible to optical frequency combs, as they constitute one of the most accurate devices available for measurements.

Consequently, we here investigate the possibility to use high-precision spectroscopy in order to measure nuclear masses and electron to nucleus mass ratios using transitions among Rydberg states of hydrogen-like ions, with a special emphasis on the example cases of ${ }^{1} \mathrm{H}$, ${ }^{2} \mathrm{H},{ }^{7} \mathrm{Li}$ and ${ }^{10} \mathrm{C}$. These masses are of great interest. The mass ratios of ${ }^{1} \mathrm{H}$ and ${ }^{2} \mathrm{H}$ are interesting for the frequency comparison of the transitions of hydrogen and anti-hydrogen which are being pursued at the moment [18], as well as for many other applications of spectroscopy in general. ${ }^{7} \mathrm{Li}$ is important for the study of solar neutrinos and for determining their masses [19]. There is currently an interesting discrepancy between the recently obtained mass measurement [20] and the value in the Atomic Mass Evaluation [21] (AME2003), with the more recent value from Ref. [20] being more precise. Thus, a measurement of the ${ }^{7} \mathrm{Li}$ mass with a completely independent method would seem worthwhile. The mass of ${ }^{10} \mathrm{C}$ meanwhile plays a role in super-allowed beta decays which are studied in order to measure CabibboKobayashi-Maskawa (CKM) matrix elements [22, 23]. The possibility of determining nuclear masses by high-precision spectroscopy has previously been mentioned in Refs. [24, 25], in the context of molecular spectrosocpy where the theoretical challenges in providing accurate predictions for mass determination appear to be much greater.

In order to determine the mass of the nucleus, we consider essentially two options. 
These are based on the elimination of variables in the theoretical expressions for the transition frequencies and can be summarized as follows. Namely, up to very high accuracy $\left(\sim 10^{-14}\right)$, the Rydberg transition frequencies in one-electron ions are determined by the following parameters: (i) the nuclear mass and (ii) the Rydberg constant. Our proposed Method I applies if at least one isotope of a given charge number has a very well known mass (of relative accuracy $\sim 10^{-10}$ ) which can be used as a reference for the determination of the masses of other isotopes. We then propose to measure the same Rydberg transition in two ionic systems corresponding to two different nuclear isotopes, one of which acts as a reference, and to solve the system of the two equations for two variables, namely the Rydberg constant and the unknown nuclear mass. Method II applies if we suppose that recent efforts in measuring an improved value of the Rydberg constant (see Refs. [16, 17, 26]) are crowned with success, and that an improved value of the Rydberg constant (of relative accuracy $10^{-13} \ldots 10^{-14}$ ) is available. In that case, the repetition of a measurement of the same or of a different Rydberg transition in a one-electron ion of a different nuclear isotope or even with a nucleus of a different charge number can directly lead to a determination of the mass of that nucleus (we essentially solve two observational equations corresponding to the two measured frequencies for the two unknowns, namely, the Rydberg constant and the nuclear mass to be determined). Method III is essentially identical to method II with the only difference being that the theoretical expression is solved for the electron to nucleus mass ratio instead of the nuclear mass.

So far, transitions in circular Rydberg states have been measured up to a relative accuracy of $2.1 \times 10^{-11}$ in an $80 \mathrm{~K}$ atomic beam of hydrogen [27, 28]. The measurement was carried out in the millimeter region and shows that it is in principle possible to conduct highprecision measurements on Rydberg states. There is no fundamental obstacle to improving the experimental accuracy of infra-red and near-optical transitions among Rydberg states of hydrogen-like ions up to the level of $10^{-14} \ldots 10^{-15}$. Using femto-second lasers and frequency combs, this level of accuracy has already been attained for the lower-lying states of hydrogen. Spectroscopy on this level of accuracy has the potential to assist in the determination of nuclear masses, as we show here. In order to carry out the current study, a supplementary calculation of the self-energy remainder function is carried out in the range of low nuclear charge numbers $Z=3,6,8$, for excited states with principal quantum number $n=5, \ldots, 8$ and orbital angular momenta $\ell=n-1$ and $\ell=n-2$.

The outline of this paper implies a discussion of QED theory in Sec. 2, with a detailed discussion of the proposal for the nuclear mass determination following in Sec. 3 . The numerical and analytic methods for the treatment of the self-energy remainder functions are described in Sec. 2.2 Numerical examples concerning the attainable accuracy for the nuclear mass determination are given in Sec. 3.2. Finally, conclusions are drawn in Sec.4.

\section{QED Calculations}

\subsection{Status of Theory}

In formulating the QED theory of Rydberg transition frequencies [11, 16, 17, 29, 30], we write the transition frequency between two Rydberg states of a one-electron atom as

$$
\nu_{1 \leftrightarrow 2}=\nu_{2}-\nu_{1}
$$

for a transition between quantum states $|1\rangle$ and $|2\rangle$. The quantities $\nu_{i}(i=1,2)$ are related to the bound-state energies as $\nu_{i}=E_{i} / h$, where $h$ is Planck's constant. We now discuss the different contributions to the bound-state frequencies $\nu_{i}$ one-by-one, recalling a number of expressions from Refs. [11, 16, 17, 29, 30] which are relevant for the current study. 
The dominant contribution arises from the Dirac energy, which we convert to a Dirac frequency $\nu_{\mathrm{D}}$. We subtract the rest mass and correct for the nuclear mass. For a state with principal quantum number $n$, total angular momentum $j$, and angular momentum $\ell$, we have the well-known result

$$
\nu_{\mathrm{D}}=\frac{R_{\infty} c}{1+r(\mathcal{N})} 2\left\{f(n, j)-1-\frac{r(\mathcal{N}) \alpha^{2}}{2[1+r(\mathcal{N})]^{2}}[f(n, j)-1]^{2}\right\},
$$

where $c$ is the speed of light, $R_{\infty}=\alpha^{2} m_{e} c / 2 h$ is the Rydberg constant, $r(\mathcal{N})=$ $m_{e} / m_{N}(\mathcal{N})$ is the mass ratio of the electron to the nuclear mass, and $\alpha$ is the fine-structure constant. In writing $m_{N}(\mathcal{N})$, we use the subscript $N$ in order to denote the nuclear mass, and reserve the argument $\mathcal{N}$ in order to differentiate a specific nucleus under investigation, e.g., $\mathcal{N}={ }^{7} \mathrm{Li}$ or $\mathcal{N}={ }^{10} \mathrm{C}$. The function $f(n, j)$ is given as

$$
f(n, j)=\left[1+\frac{(Z \alpha)^{2}}{\left(n-j-\frac{1}{2}+\sqrt{\left(j+\frac{1}{2}\right)^{2}-(Z \alpha)^{2}}\right)^{2}}\right]^{-\frac{1}{2}} .
$$

For non- $S$ states, there is a further nuclear-mass dependent contribution from the socalled Barker-Glover (BG) term which originates from the two-body Breit Hamiltonian and eliminates the $(n, j)$ degeneracy of Dirac theory [31],

$$
\begin{aligned}
\nu_{\mathrm{BG}}= & \frac{R_{\infty} c}{1+r(\mathcal{N})} \frac{r(\mathcal{N})^{2} Z^{4} \alpha^{2}}{n^{3}[1+r(\mathcal{N})]^{2}} \\
& \times\left(\frac{1}{j+\frac{1}{2}}-\frac{1}{\ell+\frac{1}{2}}\right)\left(1-\delta_{\ell 0}\right) .
\end{aligned}
$$

The relativistic-recoil (RR) correction changes the frequency of a level with $\ell \geq 2$ by [32, 33, 34, 35]

$$
\begin{aligned}
& \nu_{\mathrm{RR}}=\frac{R_{\infty} c}{1+r(\mathcal{N})} \frac{2 r(\mathcal{N}) Z^{5} \alpha^{3}}{\pi n^{3}}\left\{\frac{1}{[1+r(\mathcal{N})]^{2}}\right. \\
& \times\left[-\frac{8}{3} \ln k_{0}(n, \ell)-\frac{7}{3} \frac{1}{\ell(\ell+1)(2 \ell+1)}\right] \\
& +\pi Z \alpha[1+r(\mathcal{N})] \\
& \left.\times\left[3-\frac{\ell(\ell+1)}{n^{2}} \frac{2}{\left(4 l^{2}-1\right)(2 \ell+3)}\right]+\ldots\right\},
\end{aligned}
$$

where $\ln k_{0}$ is the (nonrelativistic) Bethe logarithm that depends on $n$ and $\ell$. The QED radiative corrections for these levels contribute

$$
\begin{aligned}
\nu_{\mathrm{QED}}= & \frac{R_{\infty} c}{1+r(\mathcal{N})} \frac{2 Z^{4} \alpha^{2}}{n^{3}}\left\{-\frac{1}{1+r(\mathcal{N})} \frac{a_{e}}{\kappa(2 \ell+1)}\right. \\
& +\frac{1}{[1+r(\mathcal{N})]^{2}} \frac{\alpha}{\pi}\left[-\frac{4}{3} \ln k_{0}(n, \ell)+\frac{32}{3}\right. \\
\times & \frac{3 n^{2}-\ell(\ell+1)}{n^{2}} \frac{(2 \ell-2) !}{(2 \ell+3) !}(Z \alpha)^{2} \ln \left(\frac{1+r(\mathcal{N})}{(Z \alpha)^{2}}\right) \\
& \left.\left.+(Z \alpha)^{2} G(Z \alpha)\right]\right\},
\end{aligned}
$$


where $\kappa=(-1)^{j-\ell+1 / 2}\left(j+\frac{1}{2}\right)$ is the Dirac angular quantum number and $a_{e}$ the electron magnetic moment anomaly. The semi-analytic expansion of the self-energy remainder function $G(Z \alpha)$, which depends on $n, \ell$ and $j$, reads

$$
\begin{aligned}
G(Z \alpha)= & A_{60}+(Z \alpha)^{2}\left[A_{81} \ln (Z \alpha)^{-2}+A_{80}+\ldots\right] \\
& +\frac{\alpha}{\pi} B_{60}+\ldots+\left(\frac{\alpha}{\pi}\right)^{2} C_{60}+\ldots
\end{aligned}
$$

Here, we use the commonly accepted notation for the QED correction terms. The letters denote the loop order, i.e., $A$ coefficients arise from one-loop diagrams, $B$ from two-loop, and $C$ from three-loop QED corrections. Vacuum polarization is negligible for Rydberg states with $n \geq 5, \ell \geq 3$ and $Z \leq 8$, on the level of $10^{-15}$ of relative accuracy for the transitions under study. So, we can restrict the discussion to the self-energy contributions to the $A$ coefficients. The first subscript of the $A$ coefficients denotes the power of $Z \alpha$, while the second denotes the power of the logarithm $\ln \left[(Z \alpha)^{-2}\right]$. To achieve an appropriate accuracy using this expansion, the $A_{60}$ coefficients have to be determined. The higher-order one-photon terms except the vacuum polarization contribution to $A_{80}$ [36], which is very small, are so far unknown. Calculations also exist for $B_{60}$ (see Ref. [37]), however only for states with $\ell \leq 5$. The three-loop term $C_{60}$ as well as the higher-order two-photon contributions not listed in Eq. (7) are unknown.

Several results for the $A_{60}$ coefficient of Rydberg states in the region $n=9, \ldots, 16$ of principal quantum numbers have recently been obtained (see Refs. [16, 17, 38]). The calculation has to be done individually for each one of the ionic states, because several logarithmic sums over the spectrum of virtual excitations ("relativistic Bethe logarithms") can only be calculated numerically. In the current investigation, a different range of principal quantum number $n=5, \ldots 8$ and orbital angular momenta $\ell=n-1$ and $\ell=n-2$ will be investigated, because the corresponding transition frequencies match the most appropriate regions for the application of frequency combs to the most interesting isotopes. We investigate the one-electron systems of lithium, carbon and oxygen. The analytic results for $A_{60}$ are compared to a nonperturbative (in $Z \alpha$ ) evaluation of the self-energy remainder function $G_{\mathrm{SE}}(Z \alpha)$, which corresponds to the entire contribution of higher-order terms to the one-loop self-energy shift for the ionic states under investigation (see Tab. 11). The function $G_{\mathrm{SE}}(Z \alpha)$ is equal to the sum of the higher-order $A$ terms, including $A_{60}$, due to the electron self-energy, as written in Eq. (7).

For nuclei with nonzero nuclear spin another contribution arises from the interaction of the nuclear spin $I$ with the total angular momentum $j$ of the electron. This leads to the known hyperfine slitting of the energy levels which is given as [39]

$$
\begin{aligned}
\nu_{\mathrm{hfs}}= & \frac{R_{\infty} c}{1+r(\mathcal{N})} \frac{Z^{3} \alpha^{2}}{n^{3}}\left\{\frac{r(\mathcal{N})}{1+r(\mathcal{N})} \frac{\kappa}{|\kappa|}\right. \\
& \times \frac{g}{(2 \kappa+1)\left(\kappa^{2}-\frac{1}{4}\right)}[F(F+1)-I(I+1)-j(j+1)] \\
& \times n^{3}|\kappa|(2 \kappa+1) \frac{2 \kappa(\gamma+n-|\kappa|)-N}{N^{4} \gamma\left(4 \gamma^{2}-1\right)}\left\{1+\frac{\alpha}{\pi} \frac{1}{4 \kappa}\right\},
\end{aligned}
$$

where $g$ is the nuclear $g$ factor, $\vec{F}=\vec{I}+\vec{j}$ the total angular momentum of the one-electron ion, $\gamma=\sqrt{\kappa^{2}-(Z \alpha)^{2}}$ and $N=\sqrt{(n-|\kappa|)^{2}+2(n-|\kappa|) \gamma+\kappa^{2}}$. The term of relative order $\alpha$ has been obtained in Ref. [40]; the generalization to arbitrary quantum numbers is found here. If necessary, the corrections to the hyperfine splitting up to relative order $\alpha(Z \alpha)^{2}$ can be calculated using a generalization of the approach of Ref. [41]. 
Having discussed all the required corrections for the energy levels of Rydberg states in one-electron ions for the purposes of our investigation, we can write down the total frequency of a specific level as the sum

$$
\nu_{i}=\nu_{\mathrm{D}}+\nu_{\mathrm{BG}}+\nu_{\mathrm{RR}}+\nu_{\mathrm{QED}} .
$$

where $i=1,2$ in the spirit of Eq. (1). We here assume that the $\nu_{\mathrm{hfs}}$ has been subtracted from the transition frequency. if necessary, the hyperfine-fine structure mixing terms can be calculated according to the approach outlined in Sec. III of Ref. [12]. However, we note that the state with the highest projection $M_{F}$ in a given manifold of total electron+nuclear angular momentum $F$ does not receive any corrections due to hyperfine-structure fine-structure mixing.

\subsection{Calculation of The Self-Energy Remainder}

The expression (6) for the QED corrections to an ionic level includes recoil corrections in the form of the electron-to-nuclear mass ratio $r(\mathcal{N})$. For the evaluation of the self-energy correction, it is much more practical to use the non-recoil approximation, which implies an infinitely heavy nucleus and therefore the limit $r(\mathcal{N}) \rightarrow 0$. In the non-recoil limit, the onephoton self-energy shift $\Delta E\left(n \ell_{j}\right)$ of principal quantum number $n$, orbital angular momentum $\ell$ and total angular momentum quantum number $j$ can be written as

$$
\begin{aligned}
& \Delta E\left(n \ell_{j}\right)=2 h R_{\infty} c \frac{\alpha}{\pi} \frac{Z^{4} \alpha^{2}}{n^{3}}\left\{A_{40}\right. \\
& \left.+(Z \alpha)^{2}\left(A_{61} \ln \left[(Z \alpha)^{-2}\right]+G_{\mathrm{SE}}(Z \alpha)\right)\right\}, \quad \ell \geq 2,
\end{aligned}
$$

where the first subscript of the $A$ coefficients denotes the order of $Z \alpha$ while the second denotes the order of the logarithm $\ln \left[(Z \alpha)^{-2}\right]$. We have

$$
\lim _{Z \alpha \rightarrow 0} G_{\mathrm{SE}}(Z \alpha)=A_{60} \text {. }
$$

The form of the expansion in Eq. (10) is valid for states with orbital angular momentum quantum number $\ell \geq 2$. The known results for $A_{40}$ and $A_{61}$ for Rydberg states read as follows,

$$
\begin{aligned}
& A_{40}=-\frac{4}{3} \ln k_{0}-\frac{1}{2 \kappa(2 \ell+1)}, \\
& A_{61}=\frac{32}{3} \frac{3 n^{2}-\ell(\ell+1)}{n^{2}} \frac{(2 \ell-2) !}{(2 \ell+3) !} .
\end{aligned}
$$

In Eq. (6), the prefactor $[1+r(\mathcal{N})]^{-3}$ as well as the $1+r(\mathcal{N})$ in the logarithm account for the reduced-mass effects. Also, we reemphasize that these results are exclusively due to the self-energy effects, and that vacuum-polarization shifts vanish for states with $\ell \geq 3$ up to the order $\alpha(Z \alpha)^{10}$.

The evaluation of $A_{60}$ proceeds via a scale-separation parameter (overlapping parameter) that is used in order to express the self-energy shift $\Delta E\left(n \ell_{j}\right)$ in terms of two contributions, one from high-energy virtual photons and one from low-energy photons whose energy is commensurate with the atomic binding energy. The overlapping parameter cancels when both contributions are added, no matter what regularization is used [38]. Methods from effective field theory can be used in order to simplify the calculation of the high-energy part drastically [42, 43, 38]. The method of evaluation has otherwise been described in detail 
in Refs. [35, 16, 17]. For Rydberg states, it becomes almost indispensable to formulate the logarithmic sums over the virtual excitations, which contribute to $A_{60}$, in terms of a discretization of the spectrum on a lattice [44]. In Table 1] we present results for the states under investigation, which are highly excited Rydberg states with principal quantum numbers $n=5, \ldots, 8$ and with the configurations $\ell=n-1$ and $\ell=n-2$, and $j=\ell \pm \frac{1}{2}$ (four states for a given $n$ ).

In columns 4-15 of Table 1, we present the results of a nonperturbative (in $Z \alpha$ ) calculation of the self-energy remainder function $G_{\mathrm{SE}}(Z \alpha)$ for one-electron ions with nuclear charge numbers $Z=3,6,8$ (lithium, carbon and oxygen), for the same states that are also relevant for the analytic calculation. Note that in view of Eq. (11), the $A_{60}$ coefficient constitutes the limit as $Z \alpha \rightarrow 0$ of a quantity which is otherwise dependent on the nuclear charge. Therefore, in Table 1, a specific value of $Z$ has to be given along with any $G_{\mathrm{SE}}$ (the value of $\alpha$ employed in the calculation is $\alpha^{-1}=137.036$ ). For Rydberg states, the dependence of $G_{\mathrm{SE}}(Z \alpha)$ on its argument is very weak, and the deviation $\left|G_{\mathrm{SE}}(Z \alpha)-A_{60}\right|$ numerically is less than $10^{-4}$ for all states and all nuclear charge numbers under investigation here. Note that the evaluation of the self-energy remainder function for Rydberg states is a highly nontrivial problem [see [45]]. In general, when treating Rydberg states both analytically as well as numerically, one has to be very careful not to terminate the sum over the angular momenta of the virtual states too early, because numerically significant contributions are due to virtual excitations with high angular momenta (displaced from the angular momentum of the reference state by one unit).

\subsection{Estimate of Theoretical Uncertainties}

In order to gauge the applicability of our method for determining nuclear masses, we have to investigate the inevitable theoretical uncertainties that affect the theoretical predictions of Rydberg transition frequencies in hydrogen-like ions. These theoretical uncertainties ultimately determine the limits of accuracy for the extraction of the nuclear masses.

Two different sources of theoretical uncertainty have to be distinguished. The first of these comes from the input parameters necessary to evaluate the theoretical expression in Sec. 2.1. For these input parameters (nuclear masses and other fundamental constants), we use the values of the fundamental constants from CODATA 2006 [11], the masses from the 2003 Atomic Mass Evaluation [21] (AME2003) and the recent measurement for the ${ }^{7} \mathrm{Li}$ mass [20] which are given in Table 2 Moreover, the experimental value for $a_{e}$ is used in the theoretical expression (6); it reads

$$
a_{e}=1.15965218073(28) \times 10^{-3}
$$

as obtained with a one-electron cyclotron [46]. We also remember that the 2006 CODATA value of the Rydberg constant (see Table 2) carries a relative accuracy of $6.6 \times 10^{-12}$ which currently limits the accuracy of theoretical predictions of the transition frequencies.

Another point concerns the uncertainty of the reference data for the nuclear masses. The masses given in Ref. [20, 21] are the atomic masses. The mass of the bare nucleus needed for our calculation can be obtained by subtracting the masses of the electrons and their binding energies as described in Ref. [47]. Useful tabulations of ionization energies can likewise be found in Ref. [47, 48].

The second source of theoretical uncertainty is due to uncalculated higher-order terms in the theoretical expressions discussed in Sec. 2.1. The uncertainties of $\nu_{\mathrm{RR}}$ and $\nu_{\mathrm{QED}}$ are estimated as follows. For the higher-order recoil terms, we use the magnitude of the last contribution on the right-hand side of Eq. (5) times $(Z \alpha) \ln \left[(Z \alpha)^{-2}\right]$. The uncertainty in 
Table 1. Values of the $A_{60}$ coefficient obtained with the analytical method compared to the numerical results for the self-energy remainder function $G_{\mathrm{SE}}(Z \alpha)$ for $n=5, \ldots, n=8$ with $\ell=n-1$ and $\ell=n-2$. The numbers in parentheses are standard uncertainties in the last figure and represent the uncertainty of the final numerical integration due to the finite number of lattice points (in the case of $A_{60}$ ), and the finite number of integration nodes for the numerical evaluation of multi-dimensional integrals (in the case of $G_{\mathrm{SE}}$ ).

\begin{tabular}{cccccccc}
\hline \hline$n$ & $\ell$ & $2 j$ & $\kappa$ & $A_{60}$ & $G_{\mathrm{SE}}(Z=3)$ & $G_{\mathrm{SE}}(Z=6)$ & $G_{\mathrm{SE}}(Z=8)$ \\
\hline 5 & 3 & 5 & 3 & $0.00240315141(5)$ & $0.00241(3)$ & $0.00243(3)$ & $0.00245(3)$ \\
5 & 3 & 7 & -4 & $0.00808701545(5)$ & $0.00809(3)$ & $0.00811(3)$ & $0.00812(3)$ \\
5 & 4 & 7 & 4 & $0.00081441471(5)$ & $0.00082(3)$ & $0.00082(3)$ & $0.00082(3)$ \\
5 & 4 & 9 & -5 & $0.00241292908(5)$ & $0.00241(3)$ & $0.00241(3)$ & $0.00242(3)$ \\
\hline 6 & 4 & 7 & 4 & $0.00082746781(5)$ & $0.00083(3)$ & $0.00083(3)$ & $0.00083(3)$ \\
6 & 4 & 9 & -5 & $0.00274825060(5)$ & $0.00275(3)$ & $0.00275(3)$ & $0.00275(3)$ \\
6 & 5 & 9 & 5 & $0.00032667627(5)$ & $0.00032(3)$ & $0.00033(3)$ & $0.00033(3)$ \\
6 & 5 & 11 & -6 & $0.00100820108(5)$ & $0.00101(3)$ & $0.00101(3)$ & $0.00101(3)$ \\
\hline 7 & 5 & 9 & 5 & $0.00032590282(5)$ & $0.00032(3)$ & $0.00033(3)$ & $0.00033(3)$ \\
7 & 5 & 11 & -6 & $0.00114160310(5)$ & $0.00114(3)$ & $0.00114(3)$ & $0.00114(3)$ \\
7 & 6 & 11 & 6 & $0.00014743922(5)$ & $0.00015(3)$ & $0.00015(3)$ & $0.00015(3)$ \\
7 & 6 & 13 & -7 & $0.00048518597(5)$ & $0.00048(3)$ & $0.00049(3)$ & $0.00049(3)$ \\
\hline 8 & 6 & 11 & 6 & $0.00014449671(5)$ & $0.00013(3)$ & $0.00015(3)$ & $0.00014(3)$ \\
8 & 6 & 13 & -7 & $0.00054593341(5)$ & $0.00054(3)$ & $0.00055(3)$ & $0.00055(3)$ \\
8 & 7 & 13 & 7 & $0.00007286141(5)$ & $0.00006(3)$ & $0.00007(3)$ & $0.00007(3)$ \\
8 & 7 & 15 & -8 & $0.00025876638(5)$ & $0.00025(3)$ & $0.00026(3)$ & $0.00026(3)$ \\
\hline \hline
\end{tabular}

$\nu_{\mathrm{QED}}$ is separated into two parts. For the unknown $A_{81}$ term in the analytic expansion, we base our estimate on the analytic approach and estimate the magnitude of the $A_{81}$ term to be equal to $A_{60}$ times $(Z \alpha)^{2} \ln \left[(Z \alpha)^{-2}\right]$. This provides for a uniform estimate which is not restricted to those states for which nonperturbative, numerical results for the self-energy remainder function are available. Based on a comparison of the results in states with $\ell \leq 5$, the magnitude of $B_{60}$ [37] is estimated to be equal to the magnitude of $4 A_{60}$. This is used as the uncertainty for this second term. These estimates are analogous to those used previously in Ref. [16].

We now illustrate, by way of example, the predictive power of QED theory for Rydberg state transitions in the infra-rad and near optical range, which are accessible to frequency comb measurements in one-electron ions. To this end, we select a two-photon transition 
Nuclear masses and spectroscopy of Rydberg states

Table 2. Fundamental constants and masses used as input parameters for the evaluation of the theoretical expression and error estimates. In parentheses, we indicate the standard uncertainty. The masses $m_{A}(\mathcal{N})$ correspond to the atomic mass of an atom (including the bound electrons) with nucleus $\mathcal{N}$. By contrast, the nuclear mass is denoted as $m_{N}(\mathcal{N})$ in this article (it excludes the mass of the bound electrons).

\begin{tabular}{lc}
\hline \hline Constant & Value \\
\hline$R_{\infty} c$ & $3.289841960361(22) \times 10^{15} \mathrm{~Hz}$ \\
$\alpha$ & $7.2973525376(50) \times 10^{-3}$ \\
$a_{e}$ & $1.15965218073(28) \times 10^{-3}$ \\
$m_{e}$ & $5.4857990943(23) \times 10^{-4} \mathrm{u}$ \\
$m_{A}\left({ }^{12} \mathrm{C}\right)$ & $12.000000000(0) \mathrm{u}$ \\
$m_{A}\left({ }^{10} \mathrm{C}\right)$ & $10.0168532(4) \mathrm{u}$ \\
$m_{A}\left({ }^{6} \mathrm{Li}\right)$ & $6.015122795(16) \mathrm{u}$ \\
$m_{A}\left({ }^{7} \mathrm{Li}\right)$ & $7.0160034256(45) \mathrm{u}$ \\
$m_{A}\left({ }^{1} \mathrm{H}\right)$ & $1.00782503207(10) \mathrm{u}$ \\
$m_{A}\left({ }^{2} \mathrm{H}\right)$ & $2.014101778040(80) \mathrm{u}$ \\
\hline \hline
\end{tabular}

Table 3. Theoretical predictions for two-photon transition frequencies in atomic hydrogen and deuterium. The transition from the initial level $|1\rangle$ with quantum numbers $n=9$, $\ell=8$, and $j=15 / 2$ to the level $|2\rangle$ with quantum numbers $n=16, \ell=10$, and $j=19 / 2$ in considered. For the upper state, the higher-order self-energy coefficient reads $A_{60}\left(n=16, \ell=10, j=\frac{19}{2}\right)=1.026705(5) \times 10^{-5}$. The individual contributions are listed in Eq. 9 .

\begin{tabular}{ccc}
\hline \hline Term & ${ }^{1} \mathrm{H} \nu(\mathrm{THz})$ & ${ }^{2} \mathrm{H} \nu(\mathrm{THz})$ \\
\hline$\nu_{\mathrm{D}}$ & $27.7492826987(2)$ & $27.7568332542(2)$ \\
$\nu_{\mathrm{BG}}$ & 0.0000000000 & 0.0000000000 \\
$\nu_{\mathrm{RR}}$ & 0.0000000000 & 0.0000000000 \\
$\nu_{\mathrm{QED}}$ & 0.0000000035 & 0.0000000035 \\
Total & $27.7492827022(2)$ & $27.7568332577(2)$ \\
\hline \hline
\end{tabular}

in hydrogen, lithium and carbon and add the theoretical contributions to the transition frequency (1) given in Sec.2.1 in order to obtain a theoretical prediction. The results are given in Table 3 for hydrogen and deuterium and in Table 4 for two lithium isotopes. In both cases we consider a two-photon transition from the state $|1\rangle$ with quantum numbers $n=9, \ell=8$, $j=15 / 2$ to a state $|2\rangle$ with quantum numbers $n=16, \ell=10, j=19 / 2$. The hydrogendeuterium isotope shift with mass numbers 1 and 2 as well as for the lithium isotopes with mass numbers 6 and 7 is being considered. The same is done in Table 5 for another twophoton transition from the state $|1\rangle$ with quantum numbers $n=13, \ell=11, j=21 / 2$ to a state $|2\rangle$ with quantum numbers $n=17, \ell=13, j=25 / 2$ in carbon isotopes. If we wish to extract the Rydberg constant from a measurement of a two-photon transition [16, 17], then we have to consider ions with very well determined and known nuclear masses. In our example 
Nuclear masses and spectroscopy of Rydberg states

Table 4. Theoretical predictions for two-photon transition frequencies in two isotopes of hydrogen-like lithium. The transition from the initial level $|1\rangle$ with quantum numbers $n=9$, $\ell=8$, and $j=15 / 2$ to the level $|2\rangle$ with quantum numbers $n=16, \ell=10$, and $j=19 / 2$ in considered. We recall that for the upper state, the higher-order self-energy coefficient reads $A_{60}\left(n=16, \ell=10, j=\frac{19}{2}\right)=1.026705(5) \times 10^{-5}$. Again, the individual contributions are listed in Eq. (9).

\begin{tabular}{lcc}
\hline \hline Term & ${ }^{6} \mathrm{Li}^{2+} \nu(\mathrm{THz})$ & ${ }^{7} \mathrm{Li}^{2+} \nu(\mathrm{THz})$ \\
\hline$\nu_{\mathrm{D}}$ & $249.8573229816(17)$ & $249.8605750963(17)$ \\
$\nu_{\mathrm{BG}}$ & 0.0000000000 & 0.0000000000 \\
$\nu_{\mathrm{RR}}$ & 0.0000000000 & 0.0000000000 \\
$\nu_{\mathrm{QED}}$ & 0.0000002829 & 0.0000002829 \\
Total & $249.8573232645(17)$ & $249.8605753793(17)$ \\
\hline \hline
\end{tabular}

Table 5. Transition frequencies for the transition from level $|1\rangle$ with quantum numbers $n=$ $13, \ell=11$, and $j=21 / 2$ to level $|2\rangle$ with quantum numbers $n=17, \ell=13$, and $j=25 / 2$ in two isotopes of hydrogen-like carbon. The self-energy remainder for the upper state is estimated based on a coefficient of $A_{60}\left(n=17, \ell=13, j=\frac{25}{2}\right)=3.76900(5) \times 10^{-6}$.

\begin{tabular}{ccc}
\hline \hline Term & ${ }^{12} \mathrm{C}^{5+} \nu(\mathrm{THz})$ & ${ }^{10} \mathrm{C}^{5+} \nu(\mathrm{THz})$ \\
\hline$\nu_{\mathrm{D}}$ & $290.9760452425(19)$ & $290.9734102736(20)$ \\
$\nu_{\mathrm{BG}}$ & 0.0000000000 & 0.0000000000 \\
$\nu_{\mathrm{RR}}$ & 0.0000000001 & 0.0000000001 \\
$\nu_{\mathrm{QED}}$ & 0.0000006216 & 0.0000006216 \\
Total & $290.9760458641(19)$ & $290.9734108953(20)$ \\
\hline \hline
\end{tabular}

cases considered above in Table 3, the relative accuracy of the electron to nucleus mass ratio is as follows:

$$
\frac{\delta r\left({ }^{1} \mathrm{H}\right)}{r\left({ }^{1} \mathrm{H}\right)}=4.3 \times 10^{-10}, \quad \frac{\delta r\left({ }^{2} \mathrm{H}\right)}{r\left({ }^{2} \mathrm{H}\right)}=4.2 \times 10^{-10} .
$$

By contrast, the relative accuracy of the nuclear masses for the example cases in Tables 4 and 5 is as follows,

$$
\begin{aligned}
\frac{\delta m_{N}\left({ }^{6} \mathrm{Li}\right)}{m_{N}\left({ }^{6} \mathrm{Li}\right)} & =2.7 \times 10^{-9}, & & \frac{\delta m_{N}\left({ }^{7} \mathrm{Li}\right)}{m_{N}\left({ }^{7} \mathrm{Li}\right)}=6.4 \times 10^{-10}, \\
\frac{\delta m_{N}\left({ }^{10} \mathrm{C}\right)}{m_{N}\left({ }^{10} \mathrm{C}\right)} & =4.0 \times 10^{-8}, & \frac{\delta m_{N}\left({ }^{12} \mathrm{C}\right)}{m_{N}\left({ }^{12} \mathrm{C}\right)} & =1.2 \times 10^{-13} .
\end{aligned}
$$

Currently, none of the given uncertainties in the mass ratios limit the final accuracy of the theoretical predictions of Rydberg state transitions (the theoretical accuracy is limited by the current value of the Rydberg constant, on a level of about $7 \times 10^{-12}$ ). However, in view of a conceivable improvement of the accuracy of the Rydberg constant in the near future, the electron to nucleus mass ratios of ${ }^{1} \mathrm{H}$ and ${ }^{2} \mathrm{H}$ as well as the nuclear masses of ${ }^{6} \mathrm{Li},{ }^{7} \mathrm{Li}$ and ${ }^{10} \mathrm{C}$ may soon become a limiting factor. Specifically, for frequency measurements better 
than $6.0 \times 10^{-14}$ for ${ }^{7} \mathrm{Li}$, the nuclear mass accuracy will become limiting, and for the other nuclei, this effect becomes relevant at a relative accuracy of about $10^{-13}$ for the frequency measurements. Conversely, frequency measurements of better accuracy allow for a better mass determination using spectroscopy. The latter aspect will be analyzed in more detail in the following.

\section{Nuclear Mass Determination}

\subsection{General Paradigms}

We intend to explore the applicability of three methods for the determination of nuclear masses via high-precision spectroscopy of Rydberg transitions in hydrogen-like ions. As already outlined in Sec. 11 one of these methods is essentially based on a very precise measurement of the isotope shift of a specific Rydberg transition for two isotopes, one of which has a very well known reference mass. We denote the nucleus with the accurately known reference mass as $\mathcal{N}_{R}$ and its mass as $m_{N}\left(\mathcal{N}_{R}\right)$, whereas the other nucleus, whose mass $m_{N}\left(\mathcal{N}_{M}\right)$ is to be determined, is denoted as $\mathcal{N}_{M}$. One measures two transition frequencies, one in the reference system $\left(\nu_{1 \leftrightarrow 2}^{R}\right)$ and one in the isotope whose mass is to be determined (denoted $\nu_{1 \leftrightarrow 2}^{M}$ ). The system of equations composed of the two measured transition frequencies $\nu_{1 \leftrightarrow 2}^{R}$ and $\nu_{1 \leftrightarrow 2}^{M}$ and QED theory can then be solved for the Rydberg constant and for the mass of the unknown isotope. This method is known as method I in the current paper.

For method II and method III, we assume that various efforts of deducing an improved value for the Rydberg constant [16, 17, 26] using a Rydberg transition in a hydrogen-like ion with a very well known nuclear mass are successful. A second measured transition frequency $\nu_{1 \leftrightarrow 2}^{R}$ in a system with an inaccurately known nuclear mass $m_{N}\left(\mathcal{N}_{M}\right)$ can directly be compared to its theoretical value. The nuclear mass $m_{N}\left(\mathcal{N}_{M}\right)$ or the electron to nucleus mass ratio $r\left(\mathcal{N}_{M}\right)$ can then be determined.

For the above methods, the following order-of-magnitude estimates are relevant: We lose about four decimals when converting the frequency measurement to a measurement of the nuclear mass. Based on a comparison to high-precision spectroscopy of lower-lying levels of atomic hydrogen [49], we assume that a reasonable target accuracy for a Rydberg state transition lies in the range of $10^{-14}$. A sufficient accuracy of a nuclear reference mass for our purposes therefore corresponds to the level of $10^{-10}$. Two Rydberg transition frequencies of relative accuracy $10^{-14}$, one of which in a reference system, can be solved for the Rydberg constant (of accuracy $10^{-14}$ ) and for the unknown nuclear mass (to be determined with an accuracy of $10^{-10}$, in the context of method I). Alternatively, if the Rydberg constant is independently known with an accuracy of $10^{-14}$ via a measurement in a reference system, then any other nuclear mass can be determined with an accuracy of $10^{-10}$ by a measurement of a different transition in a different hydrogen-like ion (the latter consideration is relevant to method II).

Let us now cast these order-of-magnitudes estimates into formulas. We first have to express the dependence of a transition frequency $\nu_{1 \leftrightarrow 2}$ on the electron to nucleus mass ratio $r(\mathcal{N})=m_{e} / m_{N}(\mathcal{N})$, where $m_{N}(\mathcal{N})$ is the mass of the bare nucleus (without the bound electrons). As can be seen from the theoretical expressions in Sec. 2.1, this dependence has a complicated functional form. E.g., in the Dirac value Eq. (2), the first term is directly proportional to the reduced mass $\mu_{r}=1 /(1+r(\mathcal{N}))$, while the second is proportional to $r(\mathcal{N}) \mu_{r}^{3}$. However, the dominant and leading dependence is simply given by the approximate proportionality of the transition frequency to the reduced mass of the system. We can thus 
define a scaled frequency $f_{1 \leftrightarrow 2}$, which is related to $\nu_{1 \leftrightarrow 2}$ by

$$
\nu_{1 \leftrightarrow 2}=R_{\infty} c \frac{1}{1+r(\mathcal{N})} f_{1 \leftrightarrow 2} .
$$

The scaled frequency $f_{1 \leftrightarrow 2}$ given by theory still carries a residual dependence on the mass ratio. For stable/long living nuclei equal in mass or heavier than lithium, known electron to nucleus mass ratios provide enough accuracy so that the residual dependence of $f_{1 \leftrightarrow 2}$ does not contribute to the uncertainty on a level required for the nuclear mass determination.

Formulas for method I: We need one isotope with a well-known nuclear mass which we will use as the reference system. Its transition frequency, mass ratio and theoretical value are labeled $\nu_{1 \leftrightarrow 2}^{R}, r\left(\mathcal{N}_{R}\right)$, and $f_{1 \leftrightarrow 2}^{R}$, respectively. Based on this reference, we want to measure the mass of the nucleus of another isotope. Its transition frequency, mass ratio and theoretical value will be labeled $\nu_{1 \leftrightarrow 2}^{M}, r\left(\mathcal{N}_{M}\right)$, and $f_{1 \leftrightarrow 2}^{M}$. By measuring the transition frequencies $\nu_{1 \leftrightarrow 2}^{R}$ and $\nu_{1 \leftrightarrow 2}^{M}$ in both isotopes, we get the system of equations

$$
\begin{aligned}
& \nu_{1 \leftrightarrow 2}^{R}=R_{\infty} c \frac{1}{1+r\left(\mathcal{N}_{R}\right)} f_{1 \leftrightarrow 2}^{R}, \\
& \nu_{1 \leftrightarrow 2}^{M}=R_{\infty} c \frac{1}{1+r\left(\mathcal{N}_{M}\right)} f_{1 \leftrightarrow 2}^{M} .
\end{aligned}
$$

We cancel the Rydberg constant and obtain

$$
\begin{aligned}
\frac{\nu_{1 \leftrightarrow 2}^{R}}{\nu_{1 \leftrightarrow 2}^{M}} & =\frac{f_{1 \leftrightarrow 2}^{R}}{f_{1 \leftrightarrow 2}^{M}} \frac{1+r\left(\mathcal{N}_{M}\right)}{1+r\left(\mathcal{N}_{R}\right)} \\
& =\frac{m_{N}\left(\mathcal{N}_{R}\right) f_{1 \leftrightarrow 2}^{R}}{m_{N}\left(\mathcal{N}_{M}\right) f_{1 \leftrightarrow 2}^{M}} \frac{m_{N}\left(\mathcal{N}_{M}\right)+m_{e}}{m_{N}\left(\mathcal{N}_{R}\right)+m_{e}} .
\end{aligned}
$$

Solving for the nuclear mass $m_{N}\left(N_{M}\right)$, we obtain

$$
\begin{aligned}
m_{N}\left(\mathcal{N}_{M}\right)= & m_{N}\left(\mathcal{N}_{R}\right) \\
& \times m_{e}\left[\frac{\nu_{1 \leftrightarrow 2}^{R} f_{1 \leftrightarrow 2}^{M}}{\nu_{1 \leftrightarrow 2}^{M} f_{1 \leftrightarrow 2}^{R}}\left(m_{N}\left(\mathcal{N}_{R}\right)+m_{e}\right)-m_{N}\left(\mathcal{N}_{R}\right)\right]^{-1} .
\end{aligned}
$$

This allows us to determine the nuclear mass of one isotope $m_{N}\left(\mathcal{N}_{M}\right)$ from a measurement of a transition frequency $\nu_{1 \leftrightarrow 2}^{M}$ in this isotope, and a reference transition frequency $\nu_{1 \leftrightarrow 2}^{R}$ in an isotope with nuclear mass $m_{N}\left(\mathcal{N}_{R}\right)$.

Formulas for method II: We have already mentioned a joint theoretical and experimental project with the National Institute of Standards and Technology (NIST), whose aim is to deduce an improved value for the Rydberg constant from transitions in Rydberg states [16, 17]. Furthermore, we also mention a project at the National Physics Laboratory (NPL) in the United Kingdom where the $2 S-8 D$ transition in hydrogen is intended to be used in order to improve the accuracy of the Rydberg constant [26]. Let us assume that one of these efforts is met with success and the uncertainty of the Rydberg constant can be reduced significantly (by at least an order of magnitude as compared to the 2006 CODATA value [11]).

This would allow to open up another possible way of measuring masses which is especially interesting for isotope systems where no mass is known well enough to serve as a reference mass. Namely, provided the Rydberg constant can be determined to good accuracy in a different ionic system, the nuclear mass $m_{N}\left(\mathcal{N}_{M}\right)$ can be obtained by solving Eq. (18) yielding

$$
m_{N}\left(\mathcal{N}_{M}\right)=m_{e}\left(\frac{f_{1 \leftrightarrow 2}^{M} R_{\infty} c}{\nu_{1 \leftrightarrow 2}^{M}}-1\right)^{-1}
$$


Numerical loss is incurred because

$$
\frac{f_{1 \leftrightarrow 2}^{M} R_{\infty} c}{\nu_{1 \leftrightarrow 2}^{M}}-1=r\left(\mathcal{N}_{M}\right)
$$

where $r\left(\mathcal{N}_{M}\right)$ is rather small $\left(\approx 10^{-4} \ldots 10^{-5}\right.$ in typical cases), whereas the two terms on the left hand side are of order unity.

This equation also allows for a determination of the electron to nucleus mass ratios which we will denote as method III in the following. In this case, we just use

$$
r\left(\mathcal{N}_{M}\right)=\frac{f_{1 \leftrightarrow 2}^{M} R_{\infty} c}{\nu_{1 \leftrightarrow 2}^{M}}-1 .
$$

in order to determine $r\left(\mathcal{N}_{M}\right)$, again with a loss in numerical significance of above four decimals.

Table 6. We list the sources for the relative uncertainties for the determination of $\delta m_{N}\left(\mathcal{N}_{M}\right) / m_{N}\left(\mathcal{N}_{M}\right)$ We explore method I for the determination of a nuclear mass (see text). The reference nucleus is $\mathcal{N}_{R}={ }^{12} \mathrm{C}$, and the nuclear mass of $\mathcal{N}_{M}={ }^{10} \mathrm{C}$ is to be measured. The transition is from state $|1\rangle \leftrightarrow|2\rangle$, where $|1\rangle$ has quantum numbers $n=13$, $\ell=11$ and $j=21 / 2$, whereas $|2\rangle$ has quantum numbers $n=17, \ell=13$ and $j=25 / 2$. The contributions to the relative uncertainty $\delta m_{N}\left(\mathcal{N}_{M}\right) / m_{N}\left(\mathcal{N}_{M}\right)$ above the horizontal line are evaluated in terms of the 2006 CODATA recommended values of the fundamental constants and do not influence the determination of $m_{N}\left(\mathcal{N}_{M}\right)$ on the level of one part in $10^{-10}$. The contributions below the horizontal line are due to the assumed experimental (spectroscopic) accuracy of the transitions.

\begin{tabular}{lc}
\hline \hline Source & $\frac{\delta m_{N}\left(\mathcal{N}_{M}\right)}{m_{N}\left(\mathcal{N}_{M}\right)}$ \\
\hline$\frac{1}{m_{N}\left(\mathcal{N}_{M}\right)} \frac{\partial m_{N}\left(\mathcal{N}_{M}\right)}{\partial m_{N}\left(\mathcal{N}_{R}\right)} \delta m_{N}\left(\mathcal{N}_{R}\right)$ & $9.6 \times 10^{-14}$ \\
$\frac{1}{m_{N}\left(\mathcal{N}_{M}\right)} \frac{\partial m_{N}\left(\mathcal{N}_{M}\right)}{\partial r\left(\mathcal{N}_{M}\right)} \delta r\left(\mathcal{N}_{M}\right)$ & $1.8 \times 10^{-13}$ \\
$\frac{1}{m_{N}\left(\mathcal{N}_{M}\right)} \frac{\partial m_{N}\left(\mathcal{N}_{M}\right)}{\partial r\left(\mathcal{N}_{R}\right)} \delta r\left(\mathcal{N}_{R}\right)$ & $1.6 \times 10^{-15}$ \\
$\frac{1}{m_{N}\left(\mathcal{N}_{M}\right)} \frac{\partial m_{N}\left(\mathcal{N}_{M}\right)}{\partial \alpha} \delta \alpha$ & $1.0 \times 10^{-15}$ \\
$\frac{1}{m_{N}\left(\mathcal{N}_{M}\right)} \frac{\partial m_{N}\left(\mathcal{N}_{M}\right)}{\partial a_{e}} \delta a_{e}$ & $8.8 \times 10^{-20}$ \\
$\frac{1}{m_{N}\left(\mathcal{N}_{M}\right)} \frac{\partial m_{N}\left(\mathcal{N}_{M}\right)}{\partial m_{e}} \delta m_{e}$ & $6.9 \times 10^{-11}$ \\
$\delta\left[f_{1 \leftrightarrow 2}^{M} / f_{1 \leftrightarrow 2}^{R}\right]$ & $1.6 \times 10^{-11}$ \\
\hline$\frac{1}{m_{N}\left(\mathcal{N}_{M}\right)} \frac{\partial m_{N}\left(\mathcal{N}_{M}\right)}{\partial \nu_{1 \leftrightarrow 2}^{R}} \delta \nu_{1 \leftrightarrow 2}^{R}$ & $1.8 \times 10^{4}\left(\frac{\delta \nu_{1 \leftrightarrow 2}^{R}}{\nu_{1 \leftrightarrow 2}^{R}}\right)$ \\
$\frac{1}{m_{N}\left(\mathcal{N}_{M}\right)} \frac{\partial m_{N}\left(\mathcal{N}_{M}\right)}{\partial \nu_{1 \leftrightarrow 2}^{M}} \delta \nu_{1 \leftrightarrow 2}^{M}$ & $1.8 \times 10^{4}\left(\frac{\delta \nu_{1 \leftrightarrow 2}^{M}}{\nu_{1 \leftrightarrow 2}^{M}}\right)$ \\
\hline \hline
\end{tabular}

\subsection{Potential of the Method}

We now illustrate the two discussed methods for the determination of nuclear masses from Rydberg transition spectroscopy in hydrogen-like ions. To this end, we keep the attained 
Nuclear masses and spectroscopy of Rydberg states

Table 7. We explore the application of method II (see text) for the determination of the mass of the $\mathcal{N}_{M}={ }^{7} \mathrm{Li}$ nucleus. The transition is $|1\rangle \leftrightarrow|2\rangle$ where $|1\rangle$ is the state with quantum numbers $n=9, \ell=8$, and $j=15 / 2$, and $|2\rangle$ has quantum numbers $n=16, \ell=10$ and $j=19 / 2$. Contributions because of theoretical input data to the relative uncertainty $\delta m_{N}\left(\mathcal{N}_{M}\right) / m_{N}\left(\mathcal{N}_{M}\right)$ of the nuclear mass are given above the horizontal line, whereas contributions due to the assumed spectroscopic measurements are given below the horizontal line.

\begin{tabular}{lc}
\hline \hline Source & $\frac{\delta m_{N}\left(\mathcal{N}_{M}\right)}{m_{N}\left(\mathcal{N}_{M}\right)}$ \\
\hline$\frac{1}{m_{N}\left(\mathcal{N}_{M}\right)} \frac{\partial m_{N}\left(\mathcal{N}_{M}\right)}{\partial r\left(\mathcal{N}_{M}\right)} \delta r\left(\mathcal{N}_{M}\right)$ & $1.5 \times 10^{-15}$ \\
$\frac{1}{m_{N}\left(\mathcal{N}_{M}\right)} \frac{\partial m_{N}\left(\mathcal{N}_{M}\right)}{\partial \alpha} \delta \alpha$ & $4.4 \times 10^{-11}$ \\
$\frac{1}{m_{N}\left(\mathcal{N}_{M}\right)} \frac{\partial m_{N}\left(\mathcal{N}_{M}\right)}{\partial a_{e}} \delta a_{e}$ & $3.6 \times 10^{-15}$ \\
$\frac{1}{m_{N}\left(\mathcal{N}_{M}\right)} \frac{\partial m_{N}\left(\mathcal{N}_{M}\right)}{\partial m_{e}} \delta m_{e}$ & $4.2 \times 10^{-10}$ \\
$\delta f_{1 \leftrightarrow 2}^{M}$ & $1.1 \times 10^{-11}$ \\
$\frac{1}{m_{N}\left(\mathcal{N}_{M}\right)} \frac{\partial m_{N}\left(\mathcal{N}_{M}\right)}{\partial \nu_{1 \leftrightarrow 2}^{M}} \delta \nu_{1 \leftrightarrow 2}^{M}$ & $1.3 \times 10^{4}\left(\frac{\delta \nu_{1 \leftrightarrow 2}^{M}}{\nu_{1 \leftrightarrow 2}^{M}}\right)$ \\
$\frac{1}{m_{N}\left(\mathcal{N}_{M}\right)} \frac{\partial m_{N}\left(\mathcal{N}_{M}\right)}{\partial R_{\infty} c} \delta R_{\infty} c$ & $1.3 \times 10^{4}\left(\frac{\delta R_{\infty} c}{R_{\infty} c}\right)$ \\
\hline \hline
\end{tabular}

experimental accuracy as a variable and show to which accuracy nuclear masses can be determined for a given assumed spectroscopic accuracy. We concentrate on transitions in isotopes of atomic hydrogen, lithium and carbon that lie in the infra-red frequency range, which is an ideal application range for frequency combs.

For this illustration, we use the atomic mass unit which is also the unit generally used in measurements in Penning traps. There the masses are determined by a comparison of cyclotron frequencies of different ions. Most atomic masses and especially the mass of the electron which is rather important for our methods are in fact known more precisely in the atomic mass system. Even though there are at the moment numerous efforts to use the atomic mass unit to define the SI unit kilogram, so far the conversion factor between the two units still carries a rather large relative uncertainty of $5.0 \times 10^{-8}$. In the determination of mass ratios (close to unity) or mass differences, the uncertainty of the conversion factors become irrelevant, and these are the quantities that one needs for applications to fundamental physics. We can thus safely work in atomic mass units.

We first discuss an example application for method I, which as we recall implies the determination of Rydberg constant and nuclear mass from an accurate measurement of two transitions in hydrogen-like ions of different isotopes. The reference system is taken as the one-electron ion of ${ }^{12} \mathrm{C}$. We recall that the ${ }^{12} \mathrm{C}$ atom serves as the definition of the atomic mass unit (u) which is defined by the relation $m_{A}\left({ }^{12} \mathrm{C}\right)=12 \mathrm{u}$. The nuclear mass $m_{N}\left({ }^{12} \mathrm{C}\right)$ is obtained from $m_{A}\left({ }^{12} \mathrm{C}\right)$ by the subtraction of the electron rest masses and the binding energies. The latter carries a theoretical uncertainty which has been discussed in [47]; yet $m_{N}\left({ }^{12} \mathrm{C}\right)$ still represents a very accurate nuclear mass standard. Even taking into account this uncertainty, the final relative uncertainty of the nuclear mass is $1.2 \times 10^{-13}$ which is 
Table 8. We explore the application of method III (see text) for the determination of the electron to nucleus mass ratio for $\mathcal{N}_{M}={ }^{2} \mathrm{H}$. The transition is $|1\rangle \leftrightarrow|2\rangle$ where $|1\rangle$ is the state with quantum numbers $n=9, \ell=8$, and $j=15 / 2$, and $|2\rangle$ has quantum numbers $n=16$, $\ell=10$ and $j=19 / 2$. The contributions to the relative uncertainty $\delta r\left(\mathcal{N}_{M}\right) / r\left(\mathcal{N}_{M}\right)$ of the electron to deuteron mass ratio due to the 2006 CODATA values for the fundamental constants and masses required for the evaluation of the theoretical expressions are given above the horizontal line. Contributions due to the assumed spectroscopic measurements are given below the horizontal line.

\begin{tabular}{lc}
\hline \hline Source & $\frac{\delta r\left(\mathcal{N}_{M}\right)}{r\left(\mathcal{N}_{M}\right)}$ \\
\hline$\frac{1}{r\left(\mathcal{N}_{M}\right)} \frac{\partial r\left(\mathcal{N}_{M}\right)}{\partial r\left(\mathcal{N}_{M}\right)} \delta r\left(\mathcal{N}_{M}\right)$ & $9.1 \times 10^{-17}$ \\
$\frac{1}{r\left(\mathcal{N}_{M}\right)} \frac{\partial r\left(\mathcal{N}_{M}\right)}{\partial \alpha} \delta \alpha$ & $1.4 \times 10^{-12}$ \\
$\frac{1}{r\left(\mathcal{N}_{M}\right)} \frac{\partial r\left(\mathcal{N}_{M}\right)}{\partial a_{e}} \delta a_{e}$ & $1.2 \times 10^{-16}$ \\
$\delta f_{1 \leftrightarrow 2}^{M}$ & $6.8 \times 10^{-33}$ \\
$\frac{1}{r\left(\mathcal{N}_{M}\right)} \frac{\partial r\left(\mathcal{N}_{M}\right)}{\partial \nu_{1 \leftrightarrow 2}^{M}} \delta \nu_{1 \leftrightarrow 2}^{M}$ & $3.7 \times 10^{3}\left(\frac{\delta \nu_{1 \leftrightarrow 2}^{M}}{\nu_{1 \leftrightarrow 2}^{M}}\right)$ \\
$\frac{1}{r\left(\mathcal{N}_{M}\right)} \frac{\partial r\left(\mathcal{N}_{M}\right)}{\partial R_{\infty} c} \delta R_{\infty} c$ & $3.7 \times 10^{3}\left(\frac{\delta R_{\infty} c}{R_{\infty} c}\right)$ \\
\hline \hline
\end{tabular}

Table 9. We explore the application of method III (see text) for the determination of the electron to nucleus mass ratio for $\mathcal{N}_{M}={ }^{1} \mathrm{H}$. The transition is $|1\rangle \leftrightarrow|2\rangle$ where $|1\rangle$ is the state with quantum numbers $n=9, \ell=8$, and $j=15 / 2$, and $|2\rangle$ has quantum numbers $n=16$, $\ell=10$ and $j=19 / 2$. Again, contributions to the relative uncertainty $\delta r\left(\mathcal{N}_{M}\right) / r\left(\mathcal{N}_{M}\right)$ of the mass ratio are separated into those caused by theoretical input data which are given above the horizontal line, and contributions due to the assumed spectroscopic measurements are given below the horizontal line.

\begin{tabular}{lc}
\hline \hline Source & $\frac{\delta r\left(\mathcal{N}_{M}\right)}{r\left(\mathcal{N}_{M}\right)}$ \\
\hline$\frac{1}{r\left(\mathcal{N}_{M}\right)} \frac{\partial r\left(\mathcal{N}_{M}\right)}{\partial r\left(\mathcal{N}_{M}\right)} \delta r\left(\mathcal{N}_{M}\right)$ & $9.3 \times 10^{-17}$ \\
$\frac{1}{r\left(\mathcal{N}_{M}\right)} \frac{\partial r\left(\mathcal{N}_{M}\right)}{\partial \alpha} \delta \alpha$ & $7.0 \times 10^{-13}$ \\
$\frac{1}{r\left(\mathcal{N}_{M}\right)} \frac{\partial r\left(\mathcal{N}_{M}\right)}{\partial a_{e}} \delta a_{e}$ & $5.8 \times 10^{-17}$ \\
$\delta f_{1 \leftrightarrow 2}^{M}$ & $1.3 \times 10^{-32}$ \\
$\frac{1}{r\left(\mathcal{N}_{M}\right)} \frac{\partial r\left(\mathcal{N}_{M}\right)}{\partial \nu_{1 \leftrightarrow 2}^{M}} \delta \nu_{1 \leftrightarrow 2}^{M}$ & $1.8 \times 10^{3}\left(\frac{\delta \nu_{1 \leftrightarrow 2}^{M}}{\nu_{1 \leftrightarrow 2}^{M}}\right)$ \\
$\frac{1}{r\left(\mathcal{N}_{M}\right)} \frac{\partial r\left(\mathcal{N}_{M}\right)}{\partial R_{\infty} c} \delta R_{\infty} c$ & $1.8 \times 10^{3}\left(\frac{\delta R_{\infty} c}{R_{\infty} c}\right)$ \\
\hline \hline
\end{tabular}

still two orders of magnitude more accurate than any measured nuclear mass. The isotope whose nuclear mass we want to determine is assumed to be the one-electron ion of ${ }^{10} \mathrm{C}$. In 
both isotopes, the same transition is employed for the mass determination. The currently best value for the atomic mass of ${ }^{10} \mathrm{C}$ is given in Table 2 and has a relative uncertainty of $4 \times 10^{-8}$. Although this mass determination to 4 parts in $10^{8}$ is very accurate on an absolute scale, we should remember that there appears to be room for improvement. For comparison, one of the most precise mass measurements has recently been conducted for ${ }^{16} \mathrm{O}$ with a relative precision of $1.1 \times 10^{-11}$ (see Ref. [50]).

In terms of our notation for the "master equation" Eq. (22) for method I, we have $\mathcal{N}_{R}={ }^{12} \mathrm{C}$ and $\mathcal{N}_{M}={ }^{10} \mathrm{C}$. We specify $|1\rangle \leftrightarrow|2\rangle$ as the same transition whose frequency (based on the current values of the fundamental constants [11]) has already been evaluated in Table 5 Above the horizontal line of Table 6 , we list the contributions to the relative uncertainty $\delta m_{N}\left(\mathcal{N}_{M}\right) / m_{N}\left(\mathcal{N}_{M}\right)$ of the measured mass of the $\mathcal{N}_{M}={ }^{10} \mathrm{C}$ due to the reference nucleus mass, due to the electron-to-nuclear mass ratios $r\left(\mathcal{N}_{M}\right)$ and $r\left(\mathcal{N}_{R}\right)$, due to the 2006 CODATA value of the fine-structure constant, due to the experimental uncertainty [46] of the electron magnetic moment anomaly $a_{e}$ which enters the theoretical expression in Eq. (6), and due to the higher-order QED terms which enter the theoretical expressions for the scaled transition frequencies $f_{1 \leftrightarrow 2}^{M}$ and $f_{1 \leftrightarrow 2}^{R}$. Our chosen transition has $j=\ell-\frac{1}{2}$ for both states involved. In general, states with $j=\ell-\frac{1}{2}$ allow for QED predictions with slightly smaller uncertainties than those with $j=\ell+\frac{1}{2}$. This is because states with $j=\ell-\frac{1}{2}$ in general have numerically smaller coefficients in the semi-analytic representation of radiative, radiative-recoil and recoil corrections as compared to states with $j=\ell+\frac{1}{2}$. This trend is expected to continue for the uncalculated higher-order terms. Our procedure for estimating the higher-order terms, which expresses the estimates in terms of multiples of known coefficients, therefore leads to slightly smaller theoretical uncertainties for the mentioned transitions as compared to a case with $j=\ell+\frac{1}{2}$.

If we assume that the experimental accuracy for the spectroscopic measurements of $\delta \nu_{1 \leftrightarrow 2}^{R}$ and $\delta \nu_{1 \leftrightarrow 2}^{M}$ is of the order of $10^{-14}$, then the by far dominant contribution to the uncertainty of such a mass determination is caused by the uncertainty in the frequency measurements. Their contribution to $\delta m_{N}\left(\mathcal{N}_{M}\right) / m_{N}\left(\mathcal{N}_{M}\right)$ is roughly four orders of magnitude larger than $\delta \nu_{1 \leftrightarrow 2}^{R} / \nu_{1 \leftrightarrow 2}^{R}$ and $\delta \nu_{1 \leftrightarrow 2}^{M} / \nu_{1 \leftrightarrow 2}^{M}$, as given below the horizontal line in Table 6. Finally, to give a numerical example, we conclude that assuming a relative uncertainty in the frequency measurements of the order of $1.4 \times 10^{-12}$ could be reached, the nuclear mass of ${ }^{10} \mathrm{C}$ could be determined with a relative uncertainty of $3.6 \times 10^{-8}$ on a level comparable to the 2006 CODATA value. We hereby add all contributing uncertainties quadratically. For completeness, we note that even if QED theory carried no uncertainty, and even if our spectroscopic accuracy were perfect, the maximum accuracy reachable with method I would still be limited by the uncertainty of the reference mass. Therefore having a reference mass with a high accuracy such as ${ }^{12} \mathrm{C}$ is recommendable in order to minimize one particularly problematic source of uncertainty.

We now discuss the application of method II, which implies a measurement of a single, specific transition in a hydrogen-like ion, with the Rydberg constant having been determined in a different system. The "master equation" for this method is Eq. (23). Here, the example we study is $\mathcal{N}_{M}={ }^{7} \mathrm{Li}$, with the transition given in Table 4. The current best value [20] for the mass of ${ }^{7} \mathrm{Li}$ has a relative uncertainty of $6.4 \times 10^{-10}$. The difference, however, to the previously accepted value for the mass of ${ }^{7} \mathrm{Li}[21]$ is 14 standard deviations or $1.1 \times 10^{-6} \mathrm{u}$. Another measurement with an independent method with a comparable accuracy to [20] would therefore be of great interest. In Table 7, we list the different sources of theoretical uncertainty for the mass determination. As evident from the entry in the fifth row of Table 7 the currently accepted value of the electron mass also is significant for the mass determination of the lithium isotope, though not the primary limiting factor. It is generally assumed that an improved value 
of the electron mass could be within reach of an improved measurement of a bound-electron $g$ factor in a low $-Z$ hydrogen-like ion [51, 52, 53, 54, 55].

If it would be possible to reach an accuracy of the frequency measurements in the range of $2 \times 10^{-14}$ for both the Rydberg determination frequency as well as the Lithium ion frequency (in the setting discussed above), then the nuclear mass of ${ }^{7} \mathrm{Li}$ could be determined with a relative uncertainty of $5.6 \times 10^{-10}$. Thereby, a similar accuracy as in the measurement of [20] could be reached.

In order to examine whether the required precision in the measurement can be reached for the discussed transitions, the ratio of the transition energy $E$ to the decay width of the line $\Gamma$ has to be considered. In [16], based on a calculation for the decay width for Rydberg states carried out in [56], this $Q$ factor has been evaluated for transitions of near circular Rydberg states from $n$ to $n-1$ as

$$
Q=\frac{E_{n}-E_{n-1}}{\Gamma_{n}+\Gamma_{n-1}}=\frac{3 n^{2}}{4 \alpha(Z \alpha)^{2}} .
$$

For the transitions considered here a complete calculation of the decay width following the description in [57] leads to the results for the $Q$ factor

$$
\begin{aligned}
& Q(\mathrm{Li})[n=12, \ell=10 \rightarrow n=9, \ell=8]=8.0 \times 10^{7}, \\
& Q(\mathrm{H})[n=12, \ell=10 \rightarrow n=9, \ell=8]=7.2 \times 10^{8}, \\
& Q(\mathrm{C})[n=17, \ell=13 \rightarrow n=13, \ell=11]=2.8 \times 10^{7} .
\end{aligned}
$$

The comparison of the $Q$ factors for hydrogen and lithium show its dependence on the nuclear charge number $Z$. Measurements in the past have been able to determine the energy of a transition within $10^{-4}$ of the width of the line [8, 58]. It would be required to enhance this to about $10^{-6}$ to reach the required accuracy for a mass determination in $\mathrm{Li}$ and to $3 \times 10^{4}$ for a mass determination in C. The attempt at NIST [16, 17] to measure transitions among Rydberg states up to an accuracy of $10^{-14}$ might requires one to develop techniques which enhance the resolution of the lines to the indicated values.

Due to the higher $Q$ values for hydrogen, the uncertainty with which the transition frequencies can be determine would be about one order of magnitude smaller. Moreover, the electron to proton or deuteron mass ratios are much larger compared to mass ratios for nuclei with higher $Z$ considered so far. Because the numerical loss is directly related to the mass ratio [see Eq. (24)], another source of uncertainty is reduced.

This leads us to the discussion of the application of method III. Here, we investigate the two cases $\mathcal{N}_{M}={ }^{1} \mathrm{H}$ and $\mathcal{N}_{M}={ }^{2} \mathrm{H}$ using the transitions in Tab. 3 with the master equation (25) of method III. The theoretical sources of uncertainty are given in Table 8, Based on Refs. [8, 58], we assume that the line can be split to at least one part in $10^{4}$. Together with the $Q$ factor this leads to a conservative estimate of the relative accuracy of the frequency measurement of $1.4 \times 10^{-13}$, which we will also use for the assumed relative uncertainty of the Rydberg constant. These uncertainties would allow to determine the electron to deuteron mass ratio with a relative uncertainty of $7.3 \times 10^{-10}$; this is comparable to the 2006 CODATA mass ratio which has a relative uncertainty of $4.2 \times 10^{-10}$ [11]. Every improvement in the resolution of the line would allow to increase the accuracy with which the mass ratio can be determined. For $\mathcal{N}_{M}={ }^{1} \mathrm{H}$, the theoretical sources of uncertainty are listed in Table 9 . We use the same conservative estimate as for ${ }^{2} \mathrm{H}$ regarding the uncertainty of the measurement of the transition frequency and the Rydberg constant. Even so, the electron to proton mass ratio could still be determined with a relative uncertainty of $3.6 \times 10^{-10}$; this would be slightly better than the current best value which has a relative uncertainty of $4.3 \times 10^{-10}$ [21]. 


\section{CONCLUSIONS}

The primary goal of the current work has been to show that high-precision spectroscopy of Rydberg states in hydrogen-like ions has a far greater potential for advances in fundamental physics than just the determination of the Rydberg constant and can be used for the determination of nuclear masses as well. This finding enhances the likely impact of a successful implementation of high-precision spectroscopy of highly excited Rydberg states of hydrogen-like ions with a low and medium nuclear charge number. In general, we can say that the basic idea of the current paper is to use a lightly bound electron (in a Rydberg state) to probe the nucleus (a "Rydberg electron trap" for the nucleus). The interaction of the nucleus to the loosely bound electron could be measured with high precision. Because this interaction is well understood, it can be used to determine the mass of the nucleus. Hyperfine effects caused by the interaction of the nuclear spin with the total angular momentum of the electron are briefly considered here in Eq. (8). If necessessary, they can be calculated to relative order $\alpha(Z \alpha)^{2}$ suing the formalism recently outlined in Ref. [41].

The theoretical predictions for the transition frequencies in Rydberg states are more precise than for lower-lying transitions, because a number of problematic effects like the nuclear-size correction are effectively suppressed. It is thus possible to devise transitions in the infra-red (see Tables 3 -7) for probing nuclear masses; these transitions combine a favourable range of frequencies for the application of optical frequency combs with small uncertainties in the theoretical QED predictions. Summarizing the theoretical calculations, we can say that with an entirely realistic relative uncertainty of $1.4 \times 10^{-13}$ for the measurement of the transitions in deuterium and hydrogen, the electron to proton mass ratio could be determined with a relative uncertainty of $3.6 \times 10^{-10}$. This would mean a small improvement over the present value with a relative uncertainty of $4.3 \times 10^{-10}$. For the electron to deuteron mass ratio, a relative uncertainty comparable to the 2006 CODATA value could be achieved.

If it were possible to improve the accuracy further and to reach a relative uncertainty of $2 \times 10^{-14}$ for the two-photon transition in hydrogen-like ${ }^{7} \mathrm{Li}$, we could improve lithium nuclear masses by more than one order of magnitude as compared to their values in the AME2003 [21] adjustment and of similar accuracy as [20]. This would potentially confirm the large difference between these two values for the ${ }^{7} \mathrm{Li}$ mass from Refs. [20, 21] by an independent method. As evident from the entry in the fifth row of Table 7 , the accuracy could be further improved with a more precise value for the electron mass whose accurate determination is currently being pursued, e.g., in precise measurements of the bound-electron $g$ factors. The mass determination of ${ }^{10} \mathrm{C}$ using the isotope shift would allow us to reach a relative uncertainty comparable to the current relative uncertainty of its mass of $4.0 \times 10^{-8}$ [21] if the transition frequency could be measured with a relative uncertainty of $1.4 \times 10^{-12}$.

As evident from the last two rows of Tables 6, 7, 8 and 9, one loses about four decimals in the determination of a nuclear mass from transition frequencies, due to inevitable numerical cancellations. For method II, this is explained in the text following Eq. (24). Nevertheless, the approach leads to very promising results. With the progress made in recent years in reducing the relative uncertainties in the frequency measurement using frequency combs (see [59] and references therein), there is justified hope for even more precise results for transition frequencies in the future. In turn, they would allow to further increase the accuracy of such a mass determination. In view of the present efforts of measuring nuclear masses with increased accuracy, it certainly would be helpful to have an independent method to check the nuclear masses determined by comparing cyclotron frequencies in Penning traps [60].

Concerning the broader impact of the current proposal, we remember that ${ }^{7} \mathrm{Li}$ is one of the primordial elements, and a more precise mass measurement may help to improve our 
understanding of the processes in the Big Bang Nucleosynthesis, as well as the reason for the low binding energy of the discussed nucleus. Moreover, ${ }^{7} \mathrm{Li}$ also plays a role in many nuclear reaction processes especially in the fusion cycle of the sun where an improved mass measurement can help to find bounds for the mass of the solar neutrino [19] and also for the determination of other masses through nuclear reactions [61]. The electron to proton and electron to deuteron mass ratios are important for many applications in spectroscopy where these two isotopes are studied very intensively. For example, a more accurate electron to proton mass ratio might be very interesting for the planned comparison of transition frequencies in hydrogen and anti-hydrogen [18, 62] in order to test $C P T$ invariance. Both mass ratios also play a role in the analysis of the hydrogen-deuterium isotope shift which is used to deduce the difference of the mean square charge radii of the proton and the deuteron [3]. Another field where precise nuclear masses are of great interest is the study of the unitarity of the CKM matrix [63, 22], where super-allowed beta decays are being investigated to determine the CKM matrix element $V_{u d}$. The ${ }^{10} \mathrm{C}$ nucleus is of particular interest in these studies [23]. A further increase in accuracy for the nuclear mass $m_{N}\left({ }^{10} \mathrm{C}\right)$ can improve the bounds on CKM non-unitarity and therefore provide a check for the standard model.

In order to provide reliable theoretical predictions for such joint experimental-theoretical efforts, we have performed analytic as well as numerical calculations of the self-energy remainder function for states with $n=5, \ldots, 8$ with $\ell=n-1$ and $\ell=n-2$, and we have also obtained values for the $A_{60}$ coefficient for these states. The theoretical predictions for the notoriously problematic self-energy remainder functions show an excellent mutual agreement. This leaves little room for any conceivable changes in the theoretical predictions due to calculational errors whose existence in complex QED calculations otherwise cannot be ruled out without extensive cross checks. The field appears to be open for experimental studies.

\section{Acknowledgments}

Insightful discussions with P. J. Mohr and J. N. Tan are gratefully acknowledged. This research has been supported by the National Science Foundation (Grant PHY- 8555454) and by a Precision Measurement Grant from the National Institute of Standards and Technology.

\section{References}

[1] Niering M, Holzwarth R, Reichert J, Pokasov P, Th Udem, Weitz M, Hänsch T W, Lemonde P, Santarelli G, Abgrall M, Laurent P, Salomon C and Clairon A 2000 Phys. Rev. Lett. 84 5496-5499

[2] Weitz M, Huber A, Schmidt-Kaler F, Leibfried D, Vassen W, Zimmermann C, Pachucki K, Hänsch T W, Julien L and Biraben F 1995 Phys. Rev. A 52 2664-2681

[3] Huber A, Th Udem, Gross B, Reichert J, Kourogi M, Pachucki K, Weitz M and Hänsch T W 1998 Phys. Rev. Lett. 80 468-471

[4] de Beauvoir B, Nez F, Julien L, Cagnac B, Biraben F, Touahri D, Hilico L, Acef O, Clairon A and Zondy J J 1997 Phys. Rev. Lett. 78 440-443

[5] Schwob C, Jozefowski L, de Beauvoir B, Hilico L, Nez F, Julien L, Biraben F, Acef O, Clairon A and Zondy J J 1999 Phys. Rev. Lett. 82 4960-4963

[6] de Beauvoir B, Nez F, Julien L, Cagnac B, Biraben F, Touahri D, Hilico L, Acef O, Clairon A and Zondy J J 1997 Phys. Rev. Lett. 78 440-443

[7] Berkeland D J, Hinds E A and Boshier M G 1995 Phys. Rev. Lett. 75 2470-2473

[8] Hagley E W and Pipkin F M 1994 Phys. Rev. Lett. 721172

[9] Lundeen S R and Pipkin F M 1986 Metrologia 229

[10] Newton G, Andrews D A and Unsworth P J 1979 Phil. Trans. R. Soc. A 290373

[11] Mohr P J, Taylor B N and Newell D B 2008 Rev. Mod. Phys. 80633 
[12] Pachucki K 1996 Phys. Rev. A 532092

[13] Pachucki K 1999 Phys. Rev. A 603593

[14] Friar J L, Martorell J and Sprung D W L 1997 Phys. Rev. A 56 4579-4586

[15] Jentschura U D, Kotochigova S, Le Bigot E O, Mohr P J and Taylor B N 2005 Phys. Rev. Lett. 95163003

[16] Jentschura U D, Mohr P J, Tan J N and Wundt B J 2008 Phys. Rev. Lett. 100160404

[17] Jentschura U D, Mohr P J, Tan J N and Wundt B J 2009 Can. J. Phys. 87 757-762

[18] Gabrielse G, Larochelle P, Le Sage D, Levitt B, Kolthammer W S, McConnell R, Richerme P, Wrubel J, Speck A, George M C, Grzonka D, Oelert W, Sefzick T, Zhang Z, Carew A, Comeau D, Hessels E A, Storry C H, Weel M and Walz J 2008 Phys. Rev. Lett. 100113001

[19] Reines F 1967 Proceedings of the Royal Society of London. Series A, Mathematical and Physical Sciences 301 $159-170$

[20] Nagy S, Fritioff T, Suhonen M, Schuch R, Blaum K, Björkhage M and Bergström I 2006 Phys. Rev. Lett. 96 163004

[21] Audi G, Wapstra A H and Thibault C 2003 Nuclear Physics A 729337 - 676

[22] Blaum K 2006 Physics Reports $4251-78$

[23] Hardy J C and Towner I S 2006 Nucl. Phys. News 16N4 11-17

[24] Koelemeij J C J, Roth B, Wicht A, Ernsting I and Schiller S 2007 Phys. Rev. Lett. 98173002

[25] Karr J P, Bielsa F, Douillet A, Pedregosa Gutierrez J, Korobov V I and Hilico L 2008 Phys. Rev. A 77063410

[26] Flowers J, Baird P, Klein H, Langham C, Margolis H and Walton B 2008 Conference on Precision Electromagnetic Measurements Digest, 2008. CPEM 2008. 40-41

[27] Kleppner D (private communicaton)

[28] Vries J C D Ph.D. thesis, MIT, 2001

[29] Eides M I, Grotch H and Shelyuto V A 2001 Phys. Rep. 342 63-261

[30] Sapirstein J and Yennie D R 1990 Theory of hydrogenic bound states Quantum Electrodynamics (Advanced Series on Directions in High Energy Physics vol 7) ed Kinoshita T (Singapore: World Scientific) pp 560 672

[31] Barker W A and Glover F N 1955 Phys. Rev. 99317

[32] Erickson G W 1977 J. Phys. Chem. Ref. Data 6831

[33] Pachucki K and Grotch H 1995 Phys. Rev. A 511854

[34] Golosov E, Elkhovskii A S, Milshtein A I and Khriplovich I B 1995 Zh. Éksp. Teor. Fiz. 107393 [JETP 80 (2), 208 (1995)]

[35] Jentschura U and Pachucki K 1996 Phys. Rev. A 54 1853-1861

[36] Wichmann E H and Kroll N M 1956 Phys. Rev. 101843

[37] Jentschura U D 2006 Phys. Rev. A 74062517

[38] Wundt B and Jentschura U D 2008 Phys. Lett. B 659 571-575

[39] Jentschura U D and Yerokhin V A 2006 Phys. Rev. A 73062503

[40] Brodsky S J and Parsons R G 1967 Phys. Rev. 163 134-146

[41] Jentschura U D and Yerokhin V A 2010 Phys. Rev. A 81012503

[42] Caswell W E and Lepage G P 1986 Phys. Lett. B 167437

[43] Jentschura U D, Czarnecki A and Pachucki K 2005 Phys. Rev. A 72062102

[44] Salomonson S and Öster P 1989 Phys. Rev. A 405559

[45] Jentschura U D, Mohr P J and Tan J N 2009 J. Phys. B in press

[46] Hannecke D, Fogwell S and Gabrielse G 2008 Phys. Rev. Lett. 100120801

[47] Mohr P J and Taylor B N 2005 Rev. Mod. Phys. 771

[48] Lide D R 2007 CRC Handbook of Chemistry and Physics, 88th Edition (Crc Handbook of Chemistry and Physics) (Boca Raton, FL: CRC)

[49] Fischer M, Kolachevsky N, Zimmermann M, Holzwarth R, Th Udem, Hänsch T W, Abgrall M, Grünert J, Maksimovic I, Bize S, Marion H, Pereira Dos Santos F, Lemonde P, Santarelli G, Laurent P, Clairon A, Salomon C, Haas M, Jentschura U D and Keitel C H 2004 Phys. Rev. Lett. 92230802

[50] Dyck R S V, Jr, Pinegar D B, Liew S V and Zafonte S L 2006 International Journal of Mass Spectrometry 251 $231-242$

[51] Verdú J, Djekić S, Stahl S, Valenzuela T, Vogel M, Werth G, Beier T, Kluge H J and Quint W 2004 Phys. Rev. Lett. 92093002

[52] Vogel M, Alonso J, Djekic S, Kluge H J, Quint W, Stahl S, Verdu J and Werth G 2005 Nuclear Instruments and Methods in Physics Research Section B: Beam Interactions with Materials and Atoms 2357 - 16

[53] Werth G, Alonso J, Beier T, Blaum K, Djekic S, Hffner H, Hermanspahn N, Quint W, Stahl S, Verd J, Valenzuela $\mathrm{T}$ and Vogel M 2006 International Journal of Mass Spectrometry $251152-158$

[54] Quint W, Nikoobakht B and Jentschura U D 2008 Pis'ma v. Zh. Éksp. Teor. Fiz. 87 36-40 [JETP Lett. 87, 30 (2008)]

[55] Quint W, Moskovkhin D L, Shabaev V M and Vogel M 2008 Phys. Rev. A 78032517

[56] Jentschura U D, Le Bigot E O, Evers J, Mohr P J and Keitel C H 2005 J. Phys. B 38 S97-S105 
[57] Bethe H A and Salpeter E E 1957 Quantum Mechanics of One- and Two-Electron Atoms (Berlin: Springer)

[58] Lundeen S R and Pipkin F M 1981 Phys. Rev. Lett. 46232

[59] Haensch T W 2006 Rev. Mod. Phys. 781297

[60] Brown L S and Gabrielse G 1986 Rev. Mod. Phys. 58 233-311

[61] Ball G C, Davies W G, Forster J S and Hardy J C 1972 Phys. Rev. Lett. 28 1069-1071

[62] Bluhm R, Kostelecký V A and Russell N 1999 Phys. Rev. Lett. 82 2254-2257

[63] Abele H et al. 2004 Eur. Phys. J. C33 1-8 (Preprint hep-ph/0312150) 